\title{
Dynamic rotor mode in antiferromagnetic nanoparticles
}

Lefmann, Kim; Jacobsen, H.; Garde, J.; Hedegard, P.; Wischnewski, A.; Ancona, S. N.; Jacobsen, Hjalte Sylvest; Bahl, Christian R.H.; Kuhn, Luise Theil

Published in:

Physical Review B Condensed Matter

Link to article, DOI:

10.1103/PhysRevB.91.094421

Publication date:

2015

Document Version

Publisher's PDF, also known as Version of record

Link back to DTU Orbit

Citation (APA):

Lefmann, K., Jacobsen, H., Garde, J., Hedegard, P., Wischnewski, A., Ancona, S. N., Jacobsen, H. S., Bahl, C. R. H., \& Kuhn, L. T. (2015). Dynamic rotor mode in antiferromagnetic nanoparticles. Physical Review $B$ Condensed Matter, 91(9), [094421]. https://doi.org/10.1103/PhysRevB.91.094421

\section{General rights}

Copyright and moral rights for the publications made accessible in the public portal are retained by the authors and/or other copyright owners and it is a condition of accessing publications that users recognise and abide by the legal requirements associated with these rights.

- Users may download and print one copy of any publication from the public portal for the purpose of private study or research.

- You may not further distribute the material or use it for any profit-making activity or commercial gain

- You may freely distribute the URL identifying the publication in the public portal 


\title{
Dynamic rotor mode in antiferromagnetic nanoparticles
}

\author{
K. Lefmann, ${ }^{*}$ H. Jacobsen, J. Garde, and P. Hedegård \\ Nanoscience Center, Niels Bohr Institute, University of Copenhagen, 2100 Copenhagen Ø, Denmark
}

A. Wischnewski

Research Center Jülich, Jülich Center for Neutron Science, D-52425 Jülich, Germany

S. N. Ancona, H. S. Jacobsen, C. R. H. Bahl, and L. Theil Kuhn

Materials Science Division, Ris $\varnothing$ DTU, Technical University of Denmark, 4000 Roskilde, Denmark

and Department of Energy Conversion and Storage, Technical University of Denmark, 4000 Roskilde, Denmark

(Received 4 October 2013; revised manuscript received 27 February 2015; published 19 March 2015)

\begin{abstract}
We present experimental, numerical, and theoretical evidence for an unusual mode of antiferromagnetic dynamics in nanoparticles. Elastic neutron scattering experiments on 8-nm particles of hematite display a loss of diffraction intensity with temperature, the intensity vanishing around $150 \mathrm{~K}$. However, the signal from inelastic neutron scattering remains above that temperature, indicating a magnetic system in constant motion. In addition, the precession frequency of the inelastic magnetic signal shows an increase above $100 \mathrm{~K}$. Numerical Langevin simulations of spin dynamics reproduce all measured neutron data and reveal that thermally activated spin canting gives rise to an unusual type of coherent magnetic precession mode. This "rotor" mode can be seen as a high-temperature version of superparamagnetism and is driven by exchange interactions between the two magnetic sublattices. The frequency of the rotor mode behaves in fair agreement with a simple analytical model, based on a high-temperature approximation of the generally accepted Hamiltonian of the system. The extracted model parameters, such as the magnetic interaction and the axial anisotropy, are in excellent agreement with results from Mössbauer spectroscopy.
\end{abstract}

DOI: 10.1103/PhysRevB.91.094421

PACS number(s): 75.75.Fk, 75.20.Ck, 75.30.Ds, 78.70.Nx

\section{INTRODUCTION}

Magnetic nanoparticles have important applications in modern technology, including magnetic data storage, ferrofluids, magnetic resonance imaging, biotechnology, and biomedicine [1,2]. In addition, the study of nanoparticle magnetism have elucidated many fundamental scientific phenomena, like thermally induced fluctuations, leading to magnetization reversal, known as superparamagnetism (SPM). The basic theory for SPM was derived by Néel and Brown [3,4], who found that the SPM relaxation time is given by an Arrhenius-like expression,

$$
\tau=\tau_{0} \exp \left(\frac{K V}{k_{\mathrm{B}} T}\right)
$$

where $\tau_{0}$ is a typical attempt frequency and $K V$ is the magnetic anisotropy barrier. Equation (1) is supported by numerous experimental studies, see, e.g., Refs. [1,2]. Below temperatures where the SPM relaxation is important, the magnetic dynamics is dominated by a coherent uniform precession mode, which can be considered as a $q=0$ spin wave. This mode gives rise to a linear decrease of the magnetization with increasing temperature [5].

The magnetic dynamics of antiferromagnetic (AFM) nanoparticles has attracted much attention, because it displays much richer intrinsic dynamics than that of ferromagnetic nanoparticles [6], and because complications due to dipolar interactions between particles can be neglected. In AFM nanoparticles with uniaxial anisotropy, the uniform precession

\footnotetext{
*1efmann@nbi.ku.dk
}

mode resembles a $q=0$ AFM spin wave, and its frequency is influenced both by the anisotropy and by the strength of the magnetic exchange interaction [7].

The technique of inelastic neutron scattering has earlier been found to be efficient to investigate the magnetic dynamics of nanoparticles, see, e.g., Refs. [8-18]. In particular, in 15-nm hematite $\left(\alpha-\mathrm{Fe}_{2} \mathrm{O}_{3}\right)$ particles, the frequency of the uniform modes was found to decrease with increasing temperature. This was ascribed to anharmonicities in the uniaxial anisotropy potential [9].

In this work, we present neutron diffraction data on 8-nm hematite particles, which show an unexpected vanishing of static magnetic order at temperatures as low as $150 \mathrm{~K}$, despite the fact that the Neel temperature of bulk hematite exceeds $900 \mathrm{~K}$. We analyze this result in the light of inelastic neutron data, which show a clear signal from the uniform magnetic mode at all temperatures studied (up to $300 \mathrm{~K}$ ) and an increase with temperature of the uniform mode frequency. We further investigate the physics of this system by a theoretical model and by numerical simulations, and we find that they lead towards the existence of a dynamic "rotor" mode in AFM nanoparticles.

\section{THE NANOPARTICLE SAMPLE}

Hematite is a common AFM mineral occurring frequently in soils as nanoparticles. The magnetic ions in hematite are $\mathrm{Fe}^{3+}(s=5 / 2)$, which are essentially arranged in a hexagonal structure. The spins are ferromagnetically ordered in the $(a, b)$ planes, with alternating directions along the $c$ direction. In bulk hematite above the Morin transition, $T_{\mathrm{M}}=263 \mathrm{~K}$, the 
spins are confined to the plane by a strong planar anisotropy, while below $T_{\mathrm{M}}$, the spins turn to point along the $c$ axis [19].

In particles smaller than $\sim 20 \mathrm{~nm}$, the Morin transition is suppressed $[19,20]$. At low temperatures, the spins align along an easy direction within the $(a, b)$ plane due to a weak axial anisotropy, presumably from the particle surface. The sublattices display a small canting from antiparallel within the plane (in bulk $\sim 0.1^{\circ}[19]$ ), due to the Dzyaloshinsky-Moria interaction. Our simulations show that this small term has little effect on the properties we investigate, and it is generally irrelevant in the following discussion. A later publication will discuss this approximation in more detail [21].

The nanoparticle sample of hematite was characterized earlier [13]. The particles are phosphate coated at the surface. Electron microscopy and x-ray diffraction show that the particles have an average diameter of $8 \mathrm{~nm}$. The phosphate layer is very thin, most likely a monolayer, but sufficient to minimize magnetic interparticle interactions [13].

\section{NEUTRON SCATTERING EXPERIMENTS}

Neutron diffraction experiment were performed at the Paul Scherrer Institute $(\mathrm{CH})$, using the two-axis powder diffractometer DMC and the triple-axis spectrometer RITA-2. The DMC experiments were performed with a wavelength of $4.2 \AA$ (4.7 meV), giving an effective energy resolution wider than $5 \mathrm{meV}$ (full width at half maximum, FWHM), while the RITA-2 experiments were performed at $4.7 \AA$ ( $3.7 \mathrm{meV})$ with an energy resolution of $0.12 \mathrm{meV}$ (FWHM).

The inelastic neutron scattering experiments were performed with RITA-2 in the monochromatic imaging mode [22,23]. These experiments were performed with a constant final energy of $3.7 \mathrm{meV}$, also here with energy resolution of $0.12 \mathrm{meV}$ (FWHM).

Quasielastic neutron experiments were performed at the backscattering spectrometer BSS at Research Center Jülich, using a neutron wavelength of $6.271 \AA$ and an energy resolution of $1.0 \mu \mathrm{eV}$ (FWHM). Of the 14 detectors of BSS, we used number 2 to 5 , corresponding to $q$-values of $1.9,1.7,1.5$, and $1.3 \AA^{-1}$, respectively. Each detector covers a $q$ range of around $0.2 \AA^{-1}$. Detectors 3,4 , and 5 correspond to the structural $(10 \overline{2})$ peak and the magnetic (101) and (003) peaks, respectively.

\section{NEUTRON SCATTERING DATA}

Figure 1 shows the results of the diffraction experiments. The AFM hematite peaks (003) and (101) are clearly visible at $q$ values of 1.37 and $1.51 \AA^{-1}$, respectively, in both data sets. However, the temperature dependence of the magnetic scattering intensity is most different in the two experiments. In the two-axis experiment, the peak intensity at $300 \mathrm{~K}$ is reduced by $10 \%$ relative to the value at $10 \mathrm{~K}$, whereas the triple-axis experiment shows a reduction of $90 \%$. The only difference between these two experiments is the energy resolution, indicating that the hematite spins become almost completely dynamic with an energy scale in the range between the respective energy resolutions of the two instruments.

These initial observations prompted us to re-analyse previously published inelastic neutron scattering data from the

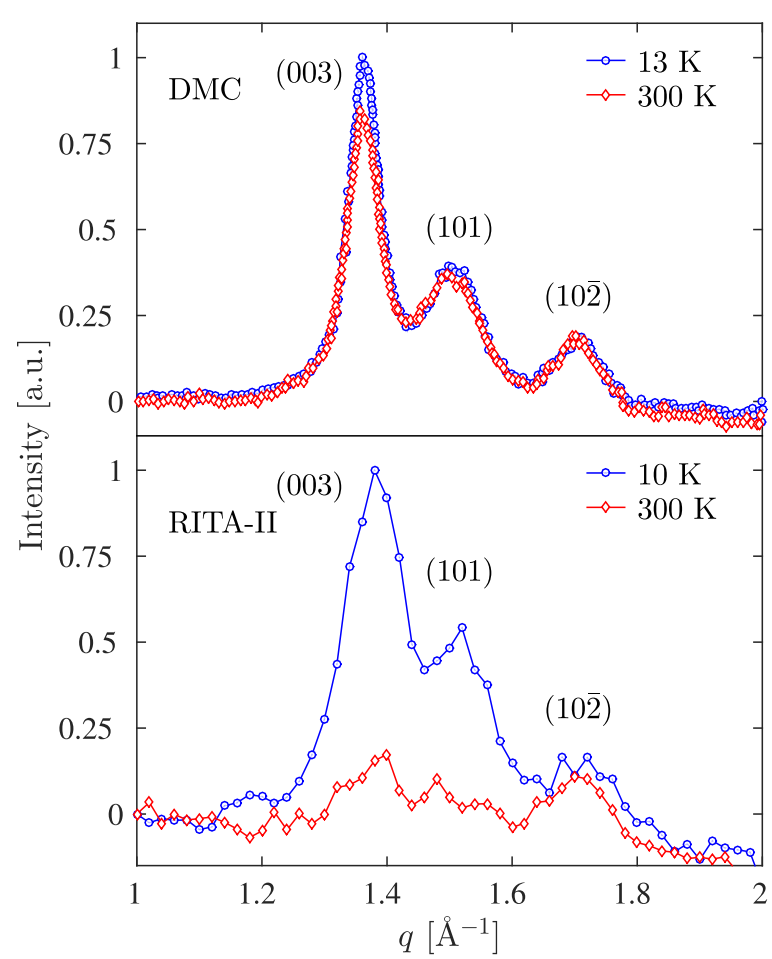

FIG. 1. (Color online) Neutron diffraction data around the magnetic (003) and (101) AFM Bragg peaks, corresponding to $q=1.37$ and $1.51 \AA^{-1}$, respectively, taken at 10 and $300 \mathrm{~K}$. (top) Data from the two-axis powder diffractometer DMC. (bottom) Data from the triple-axis spectrometer RITA-2, using energy analysis of the scattered neutrons.

same sample in the temperature range 10-200 K [13], as well as unpublished data up to $300 \mathrm{~K}$. Figure 2 shows typical data, displaying the signal at a scattering vector of $q=1.37 \AA^{-1}$, corresponding to the AFM (003) reflection. The strongest feature is the central elastic peak that stems from elastic incoherent and quasielastic background and a narrow quasielastic SPM signal.

The uniform modes are seen in the data as broad side peaks at $\left|\hbar \omega_{\alpha}\right| \sim 0.3 \mathrm{meV}$. We model these peaks by a damped harmonic oscillator (DHO) function $[9,12]$. Before correcting for experimental resolution, the model reads

$$
\begin{aligned}
I(\omega)= & A_{\mathrm{inc}} \delta(\omega)+C+\frac{A_{\mathrm{qe}} \Gamma_{\mathrm{qe}} / \pi}{\Gamma_{\mathrm{qe}}^{2}+\omega^{2}}+\frac{A_{\mathrm{SPM}} \Gamma / \pi}{\Gamma^{2}+\omega^{2}} \\
& +\frac{2 A_{\mathrm{DHO}} D(\omega) \omega_{\alpha}^{2} \gamma_{\alpha} / \pi}{\left(\omega^{2}-\omega_{\alpha}^{2}\right)^{2}+4 \gamma_{\alpha}^{2} \omega^{2}},
\end{aligned}
$$

where $C$ is a constant background, $\hbar \omega$ is the neutron energy transfer, $A_{\text {inc }}, A_{\text {qe }}, A_{\mathrm{SPM}}$, and $A_{\mathrm{DHO}}$ are the integrated intensities of the incoherent elastic, the quasielastic incoherent (from mobile $\mathrm{H}_{2} \mathrm{O}$ ), the quasielastic magnetic (from SPM), and the magnetic DHO components, respectively. $\Gamma_{\mathrm{qe}}, \Gamma$, and $\gamma_{\alpha}$ are the widths (half width at half maximum, HWHM) of the quasielastic incoherent, the quasielastic magnetic, and the inelastic peaks, respectively. $D(\omega)=\omega[n(\omega)+1] /\left[k_{\mathrm{B}} T\right]$ is the detailed balance factor [9], with $n(\omega)$ being the Bose factor and $k_{\mathrm{B}}$ being the Boltzmann constant, as detailed in [13]. The values of $A_{\mathrm{inc}}, A_{\mathrm{qe}}$, and $\Gamma_{\mathrm{qe}}$ are found from constant- $q$ scans at 

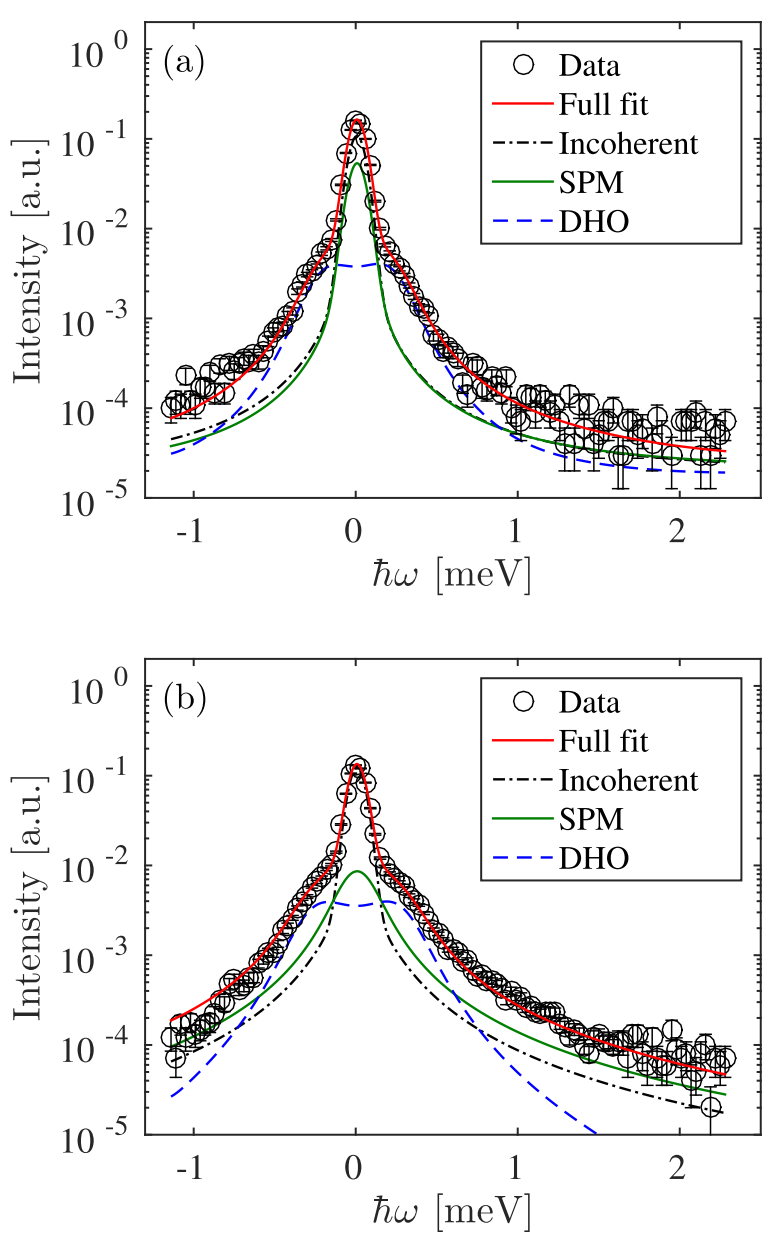

FIG. 2. (Color online) Inelastic neutron scattering data at $q=$ $1.37 \AA^{-1}$, corresponding to the (003) AFM Bragg peak. measured at $50 \mathrm{~K}$ (top) and $150 \mathrm{~K}$ (bottom). Counts are normalized to monitor and plotted on logarithmic axis vs energy transfer, $\hbar \omega$. The solid red lines show the best fit to the model (2) convoluted with experimental broadening. Individual components of the fits are shown by dot-dashed black lines, solid green lines, and dashed blue lines, which represent the incoherent elastic background, the quasielastic SPM signal, and the DHO component, respectively.

nonmagnetic $q$ values, leaving seven free fitting parameters for the magnetic scattering: $A_{\mathrm{SPM}}, \Gamma, A_{\mathrm{DHO}}, \omega_{\alpha}, \gamma_{\alpha}, C$ and a possible offset of the elastic line due to imprecision in alignment.

Experimental data taken at our lowest temperature, $10 \mathrm{~K}$, have intrinsically low signal-to-noise ratio. The parameter values for the inelastic signal then becomes unreliable, since the fit tends to model non-Gaussian tails in the resolution function for the large elastic signal, rather than the tiny inelastic magnetic signal. It was therefore necessary to disregard data taken at this temperature in the data analysis.

The quality of the fit of the model (2) to data is crucial to this work. Some correlation could be expected between the free parameters. For the important DHO signal, two parameters are of the same energy scale of a few tenths of a meV: $\gamma_{\alpha}$ and $\omega_{\alpha}$, and we have therefore investigated how these two parameters correlate by fixing their values and performing a fit of the other five parameters. Then, we record the goodness of fit given by the value of the unreduced chi-square function, $\chi_{\text {unred }}^{2}$. We have

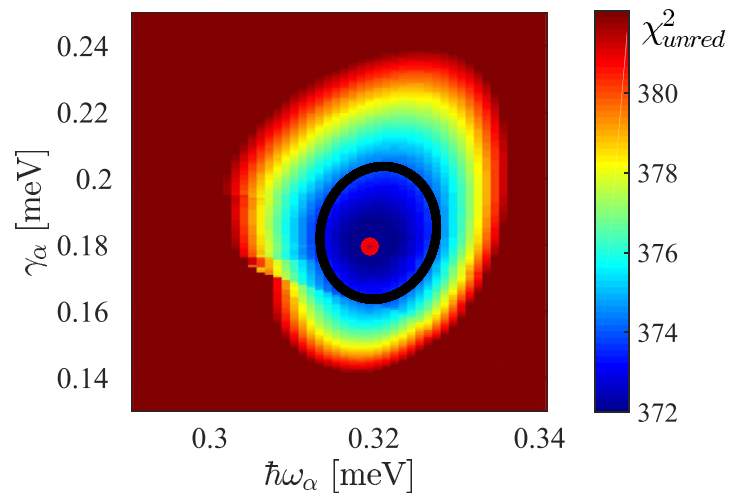

FIG. 3. (Color online) The quality of the model fit to the inelastic data from RITA-II at $150 \mathrm{~K}$, measured as the unreduced chi-square value, $\chi_{\text {unred }}^{2}$, shown for the parameter plane spanned by $\omega_{\alpha}$ and $\gamma_{\alpha}$. At each point in this plane, the value of the five other free fitting parameters have been fitted to the 115 data points. The $95 \%$ confidence interval, corresponding to $\Delta \chi_{\text {unred }}^{2}=2.3$, is marked by the solid black line.

done this for a range of parameters, spanning the $\left(\gamma_{\alpha}, \omega_{\alpha}\right)$ plane with the results presented in Fig. 3. The confidence interval in such a figure is given by the condition [24] $\Delta \chi_{\text {unred }}^{2}<2.3$ and is marked by the black solid line in the figure. We see that the values of the two parameters are essentially uncorrelated and that the fitted parameters are robust; except that the parameters may have a somewhat asymmetric errorbar.

Our central finding of experiments and model fit, the energy of the uniform mode $\hbar \omega_{\alpha}$, is shown in Fig. 4. $\omega_{\alpha}$ is found to increase with temperature, in direct contrast to the results from 15-nm particles $[9,17]$.

The width of the central peak was further studied by highresolution quasielastic measurements around the magnetic (003) peak, addressing superparamagnetic fluctuations. One example of the raw data is presented in Fig. 5 (top) together with a model fit that contains a Lorentzian line shape convoluted with the resolution function, plus a constant background. In Fig. 5 (bottom), the Lorentzian linewidths are plotted as a function of temperature. The width is seen to increase with temperature up to around $150 \mathrm{~K}$, where the magnetic signal becomes wider than the instrument energy window and no useful information can be extracted. The data up to $150 \mathrm{~K}$ is well modeled by the Neèl-Brown law (1), giving an anisotropy value of $K V / k_{\mathrm{B}}=194(40) \mathrm{K}$ with $\tau_{0}=9(2) \times 10^{-12} \mathrm{~s}$.

The remainder of this article is devoted to the explanation and modeling of the two unforeseen results: The vanishing of the diffraction peak at room temperature in the triple-axis experiments and the increase of precession frequency with temperature. In the discussions, we will also include the quasielastic neutron scattering data.

\section{ANALYTICAL THEORY}

A derivation for the spectrum of a fluctuating ferromagnetic nanoparticle in a uniaxial anisotropy was earlier derived by Würger [25]. However, in this scenario, the frequency of the uniform modes decrease with increasing temperature, in contrary to our observations. For this reason, we decided to 

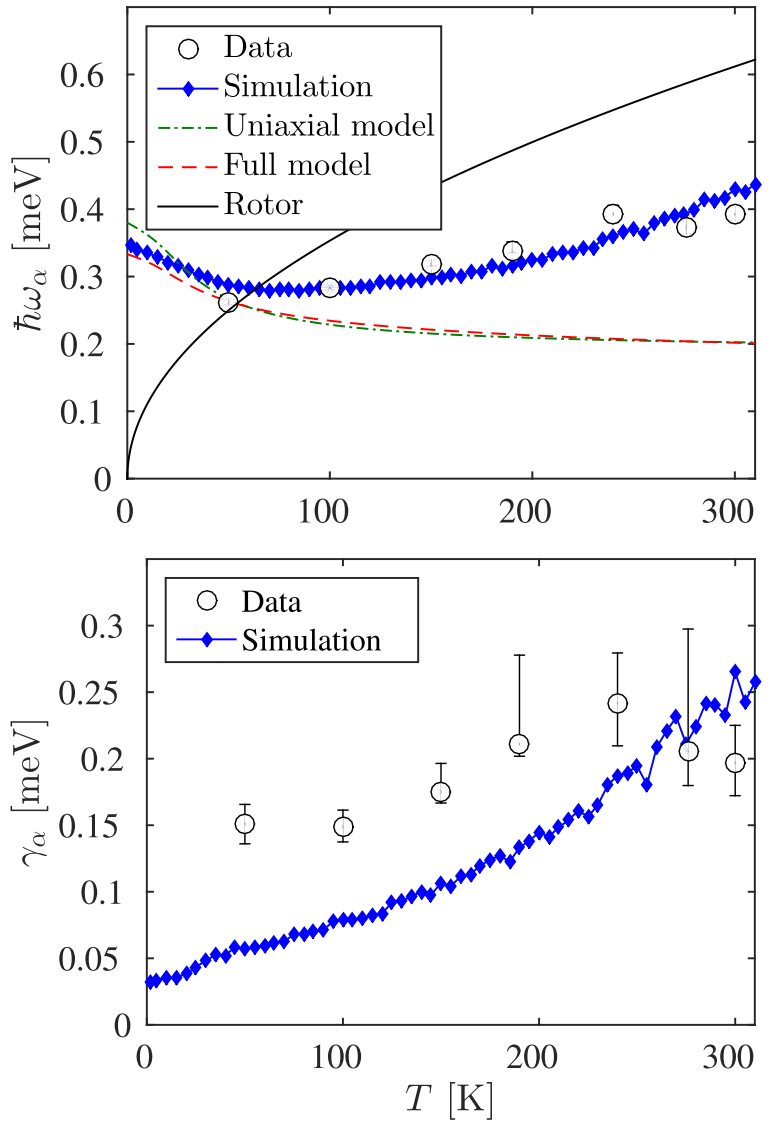

FIG. 4. (Color online) Position $\left(\omega_{\alpha}\right)$ (top), and width $\left(\gamma_{\alpha}\right)$ (bottom) of the inelastic DHO signal as a function of temperature, all obtained through Eq. (2). Black circles are measured values and small blue diamonds are results from the Langevin simulations, described in Sec. VI. Error bars on the simulated data points are comparable to the symbol sizes. In the top panel, the dot-dashed green curve in the $\hbar \omega_{\alpha}$ plot is the previously published result for the uniform mode [9], the dashed red line is the more accurate version of the same calculation [26], both without taking the "rotor" mode into account. The solid black curve is our analytical result for the rotor mode, Eq. (14).

develop an analytical understanding for this AFM system with two anisotropies.

The generally accepted microscopic magnetic Hamiltonian for hematite nanoparticles reads [7]:

$$
\mathcal{H}=\sum_{i j} J_{i j} \mathbf{s}_{i} \cdot \mathbf{s}_{j}-\kappa_{1} \sum_{i}\left(s_{i}^{x}\right)^{2}-\kappa_{2} \sum_{i}\left(s_{i}^{z}\right)^{2},
$$

where $i$ and $j$ are atomic indices, $\kappa_{1}<0$ is a large planar anisotropy, and $\kappa_{2}>0$ is a smaller axial anisotropy, which is linked to the anisotropy barrier in Eq. (1) through $K V / k_{\mathrm{B}}=$ $\kappa_{2} s s^{\prime} N$, where $s^{\prime}=s-1 / 2$ [27]. $J_{i j}$ is the exchange coupling constants. In this notation, $y$ and $z$ lie within the basal hematite $(a, b)$ plane, while $x$ is along the $c$ axis.

To describe the low-energy dynamics, we follow Würger [25] and assume collective motion $(q=0)$ of the spins in each of the two sublattices (A and B). This is justified by the fact that the highest temperature in our study, $300 \mathrm{~K}$, is far below the Néel temperature of the system and therefore spin
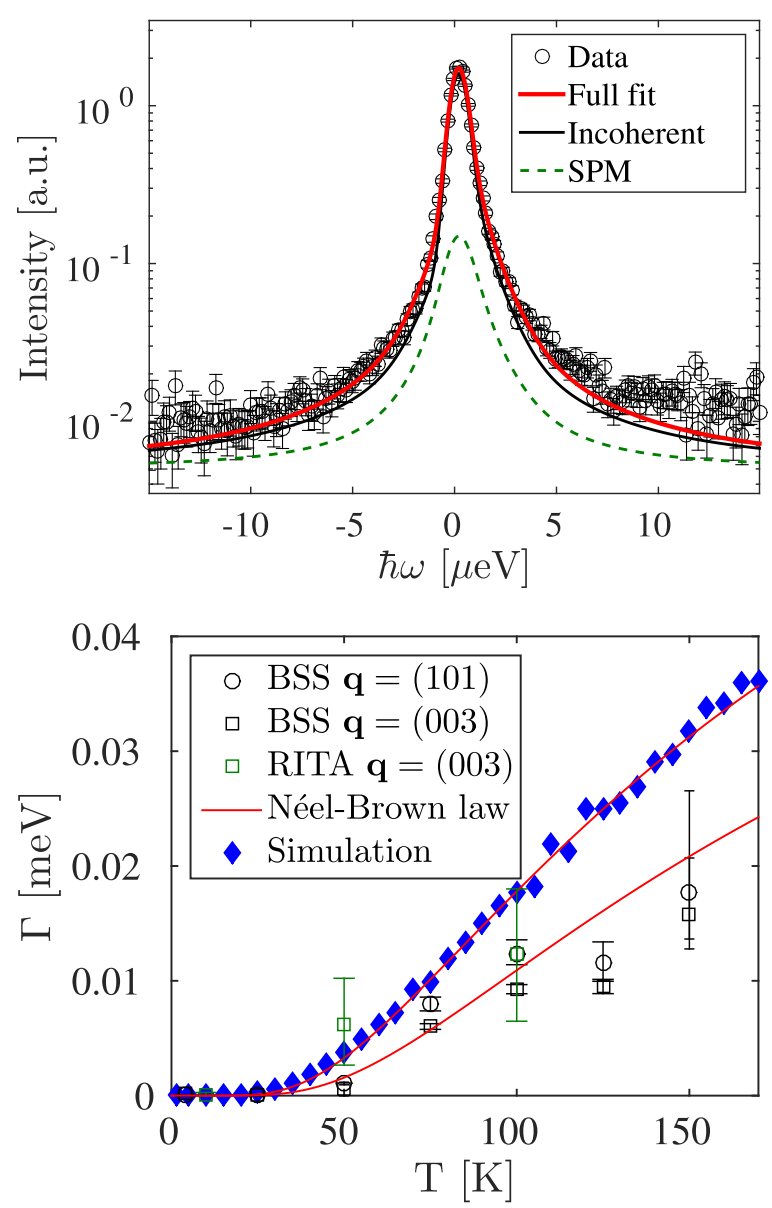

FIG. 5. (Color online) quasielastic neutron scattering data from BSS, Jülich, taken close to the magnetic (003) AFM Bragg peak. (top) Raw data taken at $T=50 \mathrm{~K}$ with a Lorentzian model fit to data, following Eq. (2). (bottom) Fitted Lorentzian line widths of the superparamagnetic signal as a function of temperature, compared with data taken at lower resolution at RITA-II, PSI, and Langevin simulations described in Sec. VI. The solid lines are fits to the NeèlBrown law, Eq. (1), as described in the text. The optimal parameters change slightly between the simulations and the experimental data, see the discussion in Sec. VII B.

waves have reduced the ordered moment of the sublattices only slightly. In addition, the finite size of the system results in a quantization of the spin wave spectrum, and due to the steepness of the hematite spin wave dispersion, only few spin waves states are in fact allowed below energies corresponding to $300 \mathrm{~K}$ [26].

We define the number of spins in the two sublattices as, $N_{A}=N_{B}$ and define the sublattice fraction $\xi=N_{A} / N_{B}$. We initially consider the case $\xi=1$, i.e., the two sublattices are equally populated, so there is no uncompensated moment. The spin dynamics can be fully described by the two superspins

$$
\mathbf{S}_{\mathrm{A}}=\hbar \sum_{i \in \mathrm{A}} \mathbf{s}_{i}, \quad \mathbf{S}_{\mathrm{B}}=\hbar \sum_{i \in \mathrm{B}} \mathbf{s}_{i} .
$$

In terms of $\mathbf{S}_{\mathrm{A}}$ and $\mathbf{S}_{\mathrm{B}}$, the Hamiltonian becomes

$$
\begin{aligned}
\mathcal{H}= & J^{\prime} \mathbf{S}_{\mathrm{A}} \cdot \mathbf{S}_{\mathrm{B}}-\kappa_{1}^{\prime}\left(S_{A}^{x 2}+S_{B}^{x 2}\right) \\
& -\kappa_{2}^{\prime}\left(S_{A}^{z 2}+S_{B}^{z 2}\right)-\gamma \mathbf{B} \cdot\left(\mathbf{S}_{\mathrm{A}}+\mathbf{S}_{\mathrm{B}}\right) .
\end{aligned}
$$


To simplify, we define $\hat{\mathbf{S}}_{\mathrm{A}}=\mathbf{S}_{\mathrm{A}} / S$, and similarly for $\hat{\mathbf{S}}_{\mathrm{B}}$. We then reach

$$
\begin{aligned}
\mathcal{H}= & \gamma S\left[B_{\mathrm{ex}} \hat{\mathbf{S}}_{\mathrm{A}} \cdot \hat{\mathbf{S}}_{\mathrm{B}}+\frac{B_{1}}{2}\left(\hat{S}_{\mathrm{A}}^{x 2}+\hat{S}_{\mathrm{B}}^{x 2}\right)\right. \\
& \left.-\frac{B_{2}}{2}\left(\hat{S}_{\mathrm{A}}^{z 2}+\hat{S}_{\mathrm{B}}^{z 2}\right)\right]
\end{aligned}
$$

where the gyromagnetic ratio is $\gamma=g \mu_{\mathrm{B}} / \hbar$ and $B_{\mathrm{ex}} \gg B_{1}>$ $B_{2}$. Here, $B_{1}$ and $B_{2}$ are the planar and uniaxial anisotropy fields, respectively, defined by $g \mu_{\mathrm{B}} B_{i}=2\left|\kappa_{i}\right| s^{\prime}$ for $i=1,2$. The exchange field is $g \mu_{\mathrm{B}} B_{\mathrm{ex}}=2 z J_{\mathrm{nn}} s$, where the factor of 2 is due to double-counting.

In bulk hematite, $B_{\mathrm{ex}}=827 \mathrm{~T}[12,19,28]$. From the neutron scattering data presented in Fig. 2 and the model Eq. (10), we find $B_{2}=4.8 \mathrm{mT}$. $B_{1}$ cannot be determined from the present neutron scattering data and we therefore use the value $B_{1}(8 \mathrm{~nm})=B_{1}(16 \mathrm{~nm})$ [17], $B_{1} \approx 46 \mathrm{mT}$ at low temperatures, gradually increasing to $\approx 66 \mathrm{mT}$ at $300 \mathrm{~K}$.

The exchange term is minimal when $\hat{\mathbf{S}}_{\mathrm{A}}$ and $\hat{\mathbf{S}}_{\mathrm{B}}$ point in opposite directions; the $B_{1}$ anisotropy is minimized if they lie in the $(y, z)$ plane, and the $B_{2}$ anisotropy will attain its minimum when $\hat{\mathbf{S}}_{\mathrm{A}}$ and $\hat{\mathbf{S}}_{\mathrm{B}}$ are parallel to the $z$ axis. Hence we have two minimum energy configurations $\hat{\mathbf{S}}_{\mathrm{A}}=(0,0, \pm 1)$, $\hat{\mathbf{S}}_{\mathrm{B}}=(0,0, \mp 1)$.

Since the magnitudes of the superspins are much larger than $\hbar$, quantum mechanics plays no role, and the dynamics is governed by the classical equations of motion:

$$
\frac{d S^{i}}{d t}=\left[S^{i}, \mathcal{H}\right],
$$

where the right-hand side is a Poisson bracket, obeying the rule $\left[S^{x}, S^{y}\right]=S^{z}$, etc. Using $\mathcal{H}$ from (6), we get

$$
\frac{d \hat{\mathbf{S}}_{\mathrm{A}}}{d t}=\gamma \hat{\mathbf{S}}_{\mathrm{A}} \times \mathbf{B}_{\mathrm{A}}, \quad \frac{d \hat{\mathbf{S}}_{\mathrm{B}}}{d t}=\gamma \hat{\mathbf{S}}_{\mathrm{B}} \times \mathbf{B}_{\mathrm{B}},
$$

where $\mathbf{B}_{\mathrm{A}}$ is the resulting field on sublattice A originating partly from the anisotropies, partly from the magnetization of sublattice B:

$$
\begin{aligned}
& \mathbf{B}_{\mathrm{A}}=-B_{\mathrm{ex}} \hat{\mathbf{S}}_{\mathrm{B}}+B_{1} \hat{S}_{\mathrm{A}}^{x} \hat{\mathbf{e}}^{x}+B_{2} \hat{S}_{\mathrm{A}}^{z} \hat{\mathbf{e}}^{z}, \\
& \mathbf{B}_{\mathrm{B}}=-B_{\mathrm{ex}} \hat{\mathbf{S}}_{\mathrm{A}}+B_{1} \hat{S}_{\mathrm{B}}^{x} \hat{\mathbf{e}}^{x}+B_{2} \hat{S}_{\mathrm{B}}^{z} \hat{\mathbf{e}}^{z},
\end{aligned}
$$

The scalar $\hat{S}_{A}^{x}$ is the projection of $\hat{\mathbf{S}}_{\mathrm{A}}$ onto the $x$ axis. Considering only motion with small deviations from the minimum energy configuration, we obtain two normal modes with frequencies [7]

$$
\begin{aligned}
& \omega_{\alpha}=\gamma \sqrt{\left(2 B_{\mathrm{ex}}+B_{1}+B_{2}\right) B_{2}} \approx \gamma \sqrt{2 B_{\mathrm{ex}} B_{2}} \\
& \omega_{\beta}=\gamma \sqrt{\left(2 B_{\mathrm{ex}}+B_{2}\right)\left(B_{1}+B_{2}\right)} \approx \gamma \sqrt{2 B_{\mathrm{ex}}\left(B_{1}+B_{2}\right)} .
\end{aligned}
$$

These are the two uniform modes calculated earlier, and also observed directly with inelastic neutron scattering $[9,12,17]$. The model can be extended to include $\xi \neq 1$, see Ref. [7].

The temperature dependence of the excitation energies at low temperatures is given by

$$
\omega_{\alpha, \beta}(T)=\omega_{\alpha, \beta}(0)\left\langle\hat{S}^{z}\right\rangle,
$$

where the ordered sublattice moment $\left\langle\hat{S}^{z}\right\rangle$ decreases monotonically with temperature, according to Boltzmann statistics $[12,17]$.
It turns out that the system supports another approximate mode, which we will denote the "rotor" mode, described in the following. We assume that the magnetic moments move slightly out of the $(y, z)$ plane, but not with strictly antiparallel sublattices, e.g.,

$$
\hat{\mathbf{S}}_{\mathrm{A}}=\left(\theta_{\mathrm{A}}, \hat{S}_{y}, \hat{S}_{z}\right) \quad \mathbf{S}_{\mathrm{B}}=\left(\theta_{\mathrm{B}},-\hat{S}_{y},-\hat{S}_{z}\right) .
$$

We first note that the canting angles described here are out of the basal plane, and therefore unrelated to the in-plane canting angle caused by the DM interaction. With our ansatz, the equations of motion become

$$
\begin{aligned}
\frac{d \theta_{\mathrm{A}}}{d t} & =\gamma B_{2} \hat{S}_{y} \hat{S}_{z}, \\
\frac{d \theta_{\mathrm{B}}}{d t} & =\gamma B_{2} \hat{S}_{y} \hat{S}_{z}, \\
\frac{d \hat{S}_{y}}{d t} & =-\gamma B_{\mathrm{ex}}\left(\theta_{\mathrm{A}}+\theta_{\mathrm{B}}\right) \hat{S}_{z}, \\
\frac{d \hat{S}_{z}}{d t} & =\gamma B_{\mathrm{ex}}\left(\theta_{\mathrm{A}}+\theta_{\mathrm{B}}\right) \hat{S}_{y},
\end{aligned}
$$
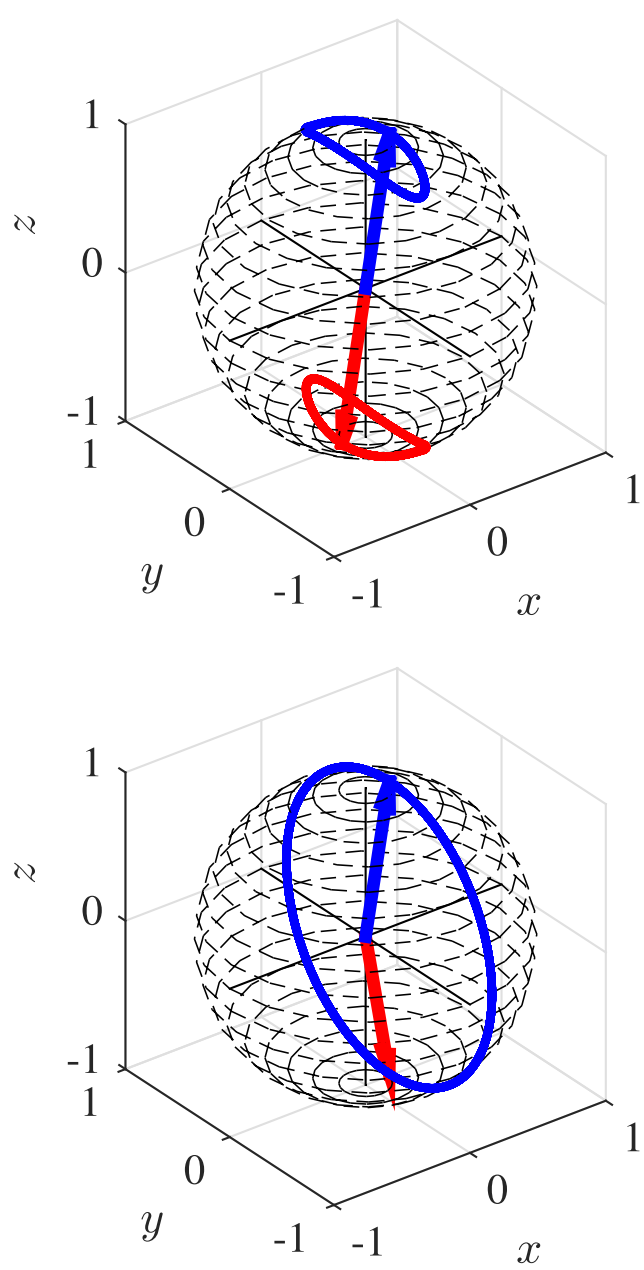

FIG. 6. (Color online) Illustration of two magnetic modes in an antiferromagnetic nanoparticle with a vertical easy axis. The A (B) sublattice points in the $+z(-z)$ direction and describes a blue (red) trajectory: (top) a uniform in-plane magnetic mode; the $\omega_{\alpha}$ mode; (bottom) the "rotor mode." 
where we neglect terms of order $B_{1}$ in comparison to terms of order $B_{\mathrm{ex}}$. Assuming that the angles $\theta_{\mathrm{A}}$ and $\theta_{\mathrm{B}}$ are constant in time, the magnetic moments will perform full $2 \pi$ rotations around the $x$ axis with a frequency given by

$$
\omega_{\text {rot }}=\gamma B_{\text {ex }}\left(\theta_{\mathrm{A}}+\theta_{\mathrm{B}}\right) \text {. }
$$

This rotation is illustrated in Fig. 6 for the case $\theta_{\mathrm{A}}=\theta_{\mathrm{B}}$. We denote the sublattice canting angle $\theta=\left|\theta_{\mathrm{A}}+\theta_{\mathrm{B}}\right|$. The $\theta$ dependence of the overall system energy stems from the exchange interaction, $E_{\theta} \propto J S^{2} \cos (\theta)$. From Boltzmann statistics, we can now calculate the thermal average value of $\theta^{2}$ to

$$
\left\langle\theta^{2}\right\rangle \approx 2 k_{B} T /\left(\gamma S B_{\mathrm{ex}}\right) .
$$

We next investigate the assumption that the relative rate of change of the angles, $\dot{\theta} / \theta$, is small compared to $\omega_{\text {rot }}$. Neglecting thermal fluctuations, we obtain

$$
\frac{\dot{\theta} / \theta}{\omega_{\text {rot }}} \approx \frac{B_{2}}{2 B_{\mathrm{ex}} \theta^{2}} \approx 5 \times 10^{-3} \frac{N_{A}}{T[K]},
$$

where we have used the values of $B_{2}$ and $B_{\text {ex }}$ given in the following. For nanoparticles corresponding to $8 \mathrm{~nm}$ hematite, $N_{A} \sim 5000$, and for $T \sim 100 \mathrm{~K}$, (16) gives $\dot{\theta} /\left(\theta \omega_{\text {rot }}\right)=$ $0.3<1$. Hence $\theta_{\mathrm{A}}$ and $\theta_{\mathrm{B}}$ maintain their values for sufficiently long times that the rotor mode is significant.

\section{NUMERICAL SIMULATIONS AND RESULTS}

To verify the soundness of the analytical approximations, and to obtain additional detail in the model, we numerically simulate the sublattice dynamics, using Langevin dynamics. Here, the equation of motion is a stochastic Landau-Lifschitz equation, very similar to the method applied earlier to describe superparamagnetism in ferromagnetic nanoparticles [29]. In the numerical calculations, we have lifted the requirement $\xi=$ 1 and also included the Dzyaloshinsky-Moriya interaction. In this scheme, the equations of motion for the two sublattices become

$$
\begin{aligned}
& \frac{d}{d t} \hat{\mathbf{S}}_{\mathrm{A}}=\gamma \hat{\mathbf{S}}_{\mathrm{A}} \times\left(\hat{\mathbf{B}}_{\mathrm{A}}+\mathbf{b}\right)-\lambda|\gamma| \hat{\mathbf{S}}_{\mathrm{A}} \times\left(\hat{\mathbf{S}}_{\mathrm{A}} \times \mathbf{B}_{\mathrm{A}}\right), \\
& \frac{d}{d t} \hat{\mathbf{S}}_{\mathrm{B}}=\gamma \hat{\mathbf{S}}_{\mathrm{B}} \times\left(\hat{\mathbf{B}}_{\mathrm{B}}+\mathbf{b}\right)-\lambda|\gamma| \hat{\mathbf{S}}_{\mathrm{B}} \times\left(\hat{\mathbf{S}}_{\mathrm{B}} \times \mathbf{B}_{\mathrm{B}}\right),
\end{aligned}
$$

where

$$
\begin{aligned}
\mathbf{B}_{\mathrm{A}}= & -\xi B_{\mathrm{ex}} \hat{\mathbf{S}}_{\mathrm{B}}+B_{1} \hat{S}_{\mathrm{A}}^{x} \hat{\mathbf{e}}^{x}+B_{2} \hat{S}_{\mathrm{A}}^{z} \hat{\mathbf{e}}^{z} \\
& -\xi B_{D}\left(\hat{S}_{B}^{z} \hat{\mathbf{e}}^{y}-\hat{S}_{B}^{y} \hat{\mathbf{e}}^{z}\right), \\
\mathbf{B}_{\mathrm{B}}= & -B_{\mathrm{ex}} \hat{\mathbf{S}}_{\mathrm{A}}+B_{1} \hat{S}_{\mathrm{B}}^{x} \hat{\mathbf{e}}^{x}+B_{2} \hat{S}_{\mathrm{B}}^{z} \hat{\mathbf{e}}^{z} \\
& +B_{D}\left(\hat{S}_{A}^{z} \hat{\mathbf{e}}^{y}-\hat{S}_{A}^{y} \hat{\mathbf{e}}^{z}\right) .
\end{aligned}
$$

$B_{D}$ is the Dzyaloshinsky-Moriya field, where we use the bulk hematite value of $B_{D}=2.1 \mathrm{~T}$ [19]. In (17), b is a small random field representing thermal fluctuations, which are isotropic and uncorrelated in time. The term $\lambda$ represents dissipation. The fluctuation-dissipation theorem [30] is used to determine in absolute units the simulated temperature:

$$
k_{\mathrm{B}} T=|\gamma| g \mu_{B} s \frac{\left\langle\mathbf{b}_{A}^{2}\right\rangle N_{A}}{2 \lambda} .
$$

Note that the particle size is present here through $N_{A}$. The fluctuating field is uncorrelated in time, $\left\langle b(t) b\left(t^{\prime}\right)\right\rangle \propto$ $\delta\left(t-t^{\prime}\right)$

The equations of motion (17) were integrated numerically using the Runge-Kutta method of second order [24] with a time step of $1 \mathrm{fs}(1000 \mathrm{THz})$, much faster than typical frequencies of the uniform mode $\omega_{\alpha}(0.5 \mathrm{THz})$. The method was tested against analytical results in the limit of vanishing fluctuation and dissipation [7,19], and for the one-sublattice (ferromagnetic) case $[27,31]$.

Examples of raw simulation data for the two-sublattice case are shown in Fig. 7. The motions of the sublattice magnetizations were, in turn, Fourier transformed to obtain the power spectra, which through the Wiener-Khintzine formula [32] yield the simulated scattering intensities. Since the scattering vector lies in the $x$ direction, we sum the power spectra of the $y$ and $z$ spin components to model experimental data. The simulated data was convoluted with the experimental resolution and fitted in the same way as the experimental data, using (2), resulting in good agreements over three orders of magnitude; see Fig. 8. The deviations found at
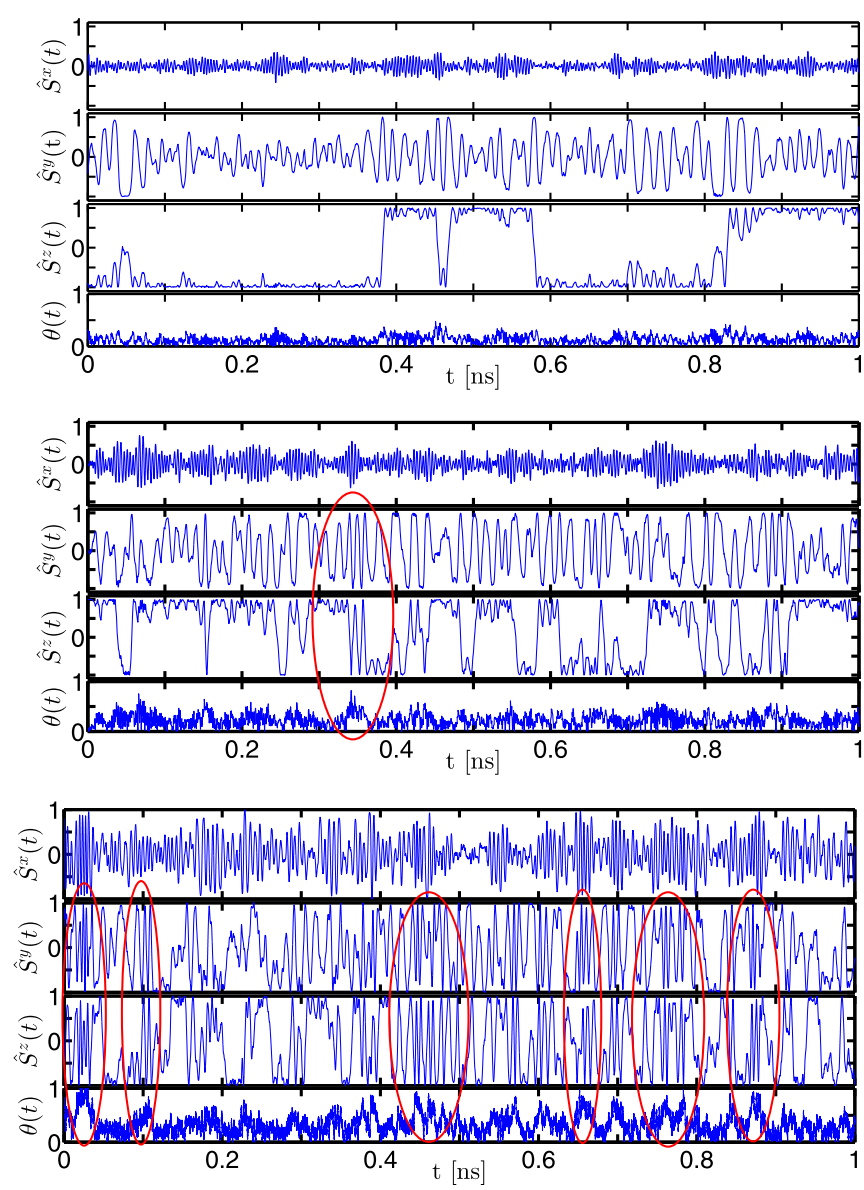

FIG. 7. (Color online) Raw data from the Langevin simulations, showing the time development of the three spin components of one superspin at temperatures of 40 (top), 150 (center), and $300 \mathrm{~K}$ (bottom). $\theta(t)$ represents the canting between the two superspins and is displayed in degrees. The encircled areas in the center and bottom panel show fast coherent oscillations in the $y z$ plane correlated with a large value of the canting angle. 

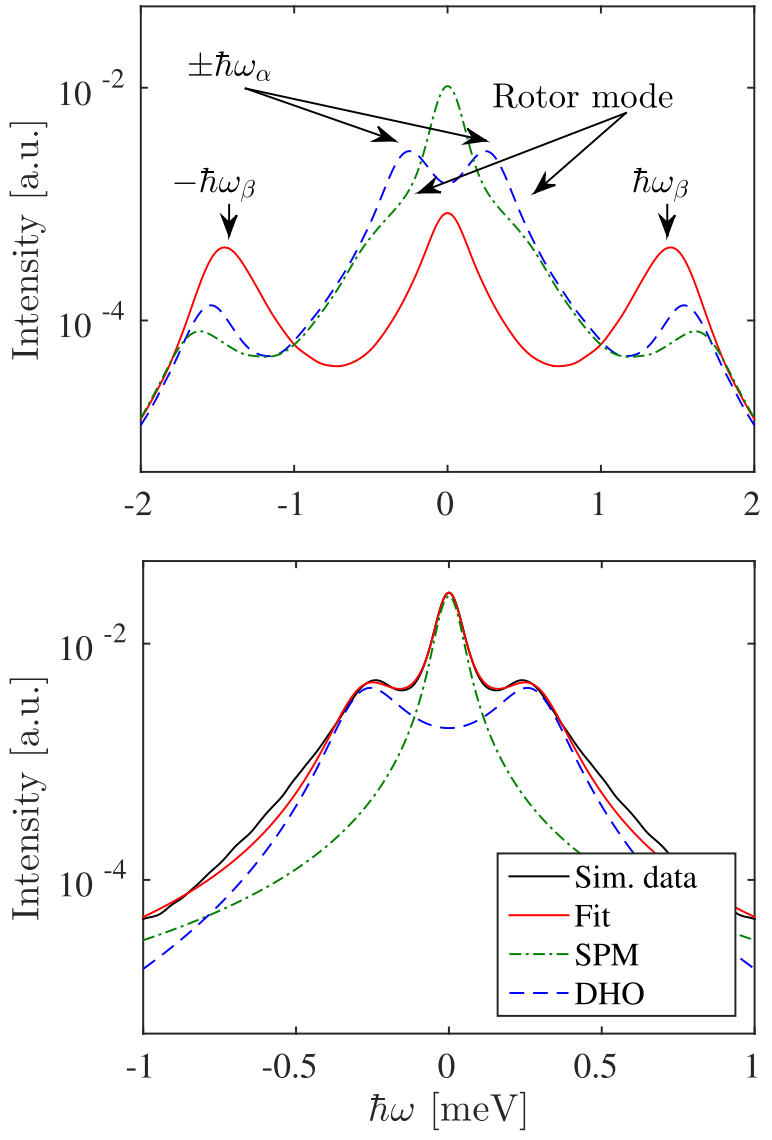

FIG. 8. (Color online) Power spectra from the Langevin simulations at $150 \mathrm{~K}$. (Top) The individual components, $\hat{S}_{x}$ (solid red), $\hat{S}_{y}$ (dashed blue), and $\hat{S}_{z}$ (dash-dotted green). All three oscillator modes are clearly visible, as indicated in the figure. (Bottom) Sum of the $y$ and $z$ components (solid black) with a fit (solid red). The individual components of the fit are the SPM and the DHO signals, given by the dash-dotted green and dashed blue lines, respectively.

the small-amplitude data at $|\hbar \omega|>0.8 \mathrm{meV}$ are irrelevant for our conclusions and we neglect them in the following.

In the simulations, we have adjusted four parameters: the uniaxial anisotropy field $B_{2}$, the exchange field $B_{\mathrm{ex}}$, the sublattice fraction, $\xi$ and the dissipation parameter $\lambda$ to find the values that yield the best agreement between simulations and experiment with respect to the temperatures dependencies of $\gamma_{\alpha}$ and $\omega_{\alpha}$ [31]. We find $B_{2}=9.5 \mathrm{mT}$, corresponding to $K V / k_{\mathrm{B}}=169 \mathrm{~K} . B_{\mathrm{ex}}$ and $\xi$ cannot be determined independently: any value of $B_{\mathrm{ex}}$ between 600 and $1000 \mathrm{~T}$ has a corresponding value of $\xi$ where simulations and data match. We therefore fix the exchange field to the bulk value, $B_{\mathrm{ex}}=827 \mathrm{~T}$, and thus find $\xi=1.010$. Finally, we determine $\lambda=6 \times 10^{-4}$.

\section{DISCUSSION}

\section{A. Existence of the rotor mode}

We first discuss our hypothesis of the existence of a rotor mode by comparing data from neutron scattering experiments, numerical simulations, and analytical calculations.
Figure 4 (top) displays the temperature dependence of the mean precession frequency, $\omega_{\alpha}$, defined through (2). The agreement on the temperature behavior of $\omega_{\alpha}$ between experiment and simulation is excellent.

The value of the DHO peak width $\gamma_{\alpha}$, displayed in Fig. 4 (bottom), is generally higher in the experimental data than in the simulations. We ascribe the higher experimental value of $\gamma_{\alpha}$ to a distribution of particle sizes and shapes, which results in a spread in $\omega_{\alpha}$. This would appear in the fit as an overall increase of $\gamma_{\alpha}$, most prominent at the lowest values of this parameter, as the particle size distribution effect is presumably constant and the width of the two broadenings should add in quadrature. This is in good overall agreement with the experimental observations.

The simulated values of $\omega_{\alpha}$ are in agreement with the neutron scattering data. At low temperatures, $T \leqslant 50 \mathrm{~K}$, the analytical uniform mode prediction agrees well with the simulations. At higher temperatures, the uniform mode prediction strongly underestimates the value of $\omega_{\alpha}$, while our analytical results for the rotor mode overestimates this value. We believe the reason for this to be the following: both the uniform mode and the rotor mode give rise to a DHO signal, which due to the relatively short life time of the excitations are both quite broad. Experimentally, we cannot resolve the two individual DHOs, and a single DHO has therefore been used to fit the data. The observed frequency is thus a weighted average of the frequency of the rotor mode, $\omega_{\text {rot }}$ and that of the uniform mode, $\omega_{\alpha}$, in general agreement with the experimental and simulated data.

Additional insight is found by inspecting the simulated time evolution of the magnetic dynamics, shown in Fig. 7. The 40-K data show low-amplitude, high-frequency oscillations in $\hat{S}^{x}$ and high-amplitude, low-frequency oscillations in $\hat{S}^{y}$, representing the two uniform modes (10). The easy-axis component, $\hat{S}^{z}$, displays nearly constant values, interrupted by sudden (SPM) magnetization reversals. These three observations are in accordance with the present understanding of magnetic dynamics of nanoparticles. The $150-\mathrm{K}$ data contain periods with much less regular motion of the sublattice spins. In particular, both $\hat{S}^{z}$ and $\hat{S}^{y}$ show oscillations. The highlighted regions show time intervals with coupled fast oscillations in these parameters, correlated with a high value of $\theta$. These fast oscillations can be seen as repeated, coherent SPM relaxations and rarely appear at the lowest temperatures. At $150 \mathrm{~K}$, they appear at least once per simulated nanosecond, and dominate at larger temperatures, e.g., at $300 \mathrm{~K}$. These events are indeed occurrences of short-lived rotor dynamics.

Our explanations are supported by the power spectra shown in Fig. 8, where the SPM behavior is found in spin components along the easy $z$ direction, the DHO signals are seen in the $y$ and $x$ components, while the rotor mode is seen as a high-frequency shoulder in the $z$ signal. The expected rotor contribution to the spectrum of $S_{y}$ is not clearly visible due to the broadening of the signal from the $\omega_{\alpha}$ mode.

\section{B. Comparison with earlier work}

In earlier work by our group, we found a value for the energy barrier $K V / k_{\mathrm{B}}=250(30) \mathrm{K}$ using the ratio between elastic and inelastic signal in the neutron scattering data from 
the same sample, and $K V / k_{\mathrm{B}}=335(35) \mathrm{K}$ using Mössbauer spectroscopy data of the same sample [13]. However, this work used an effective 3D model, in which the in-plane anisotropy is neglected, leading to an overestimation of $K V$. Assuming that the in-plane anisotropy is identical to the value for $16 \mathrm{~nm}$ particles [17], and that $\xi=1$, the values become $K V / k_{\mathrm{B}}=148(18) \mathrm{K}$ from the neutron data and $K V / k_{\mathrm{B}}=211(20) \mathrm{K}$ from the Mössbauer data. From the values of $\omega_{\alpha}$, we furthermore found a sublattice fraction of $\xi=1.010$ using the uniaxial model.

From the fit of the backscattering data to the Neèl-Brown model, we find in the present work a value of the energy barrier, $K V$ of 194(40) K, in agreement with the Mössbauer data. In addition we obtain the prefactor value $\tau_{0}=9(2) \times 10^{-12} \mathrm{~s}$.

Tuning the simulations to obtain best agreement between the measured and simulated values of $\hbar \omega_{\alpha}$ and $\gamma_{\alpha}$, we reach a "modeled refinement" of the neutron data, giving $K V / k_{\mathrm{B}}=169 \mathrm{~K}$ and $\xi=1.010$, in very good agreement with earlier work. The simulated backscattering data using the same simulation parameters also follow the Néel-Brown law very closely, leading to $K V / k_{\mathrm{B}}=169 \mathrm{~K}$, but with a slightly smaller prefactor value, $\tau_{0}^{\text {sim }}=6.8 \times 10^{-12} \mathrm{~s}$. Hence our data and simulations show a very good internal consistency, except for the $\tau_{0}$ value. This relatively minor difference, we ascribe to anharmonicities in the anisotropy potential, not included in the simulations.

\section{Uncompensated moment and coupling of modes}

Contrary to expectations from simple theory, our simulations show coupling between the $\omega_{\alpha}$ and $\omega_{\beta}$ modes. This is most obvious in the power spectra in Fig. 8, where the $x$-component has a broad central peak. At lower temperatures, this signal consists of two peaks at $\pm \hbar \omega_{\alpha}$; the peaks gradually become broader and merge as the temperature is increased. This signal is therefore caused by a weak coupling of the $\omega_{\alpha}$ and the $\omega_{\beta}$ modes. We observe no signs that the rotor mode is coupled to these mode or is changed by this weak coupling. By varying the simulation parameters, we found the coupling to be due to the sublattice fraction. At $\xi=1$, the modes seem to be completely uncoupled as expected. However, our simulations with $\xi=1.010$ in our view represents closer the physical reality for a typical nanoparticle, and it is satisfying that even with this complication we are able to reproduce the experimental observations so accurately.

\section{Absence of elastic scattering}

As a final test of the rotor model, we return to the initial observation of the disappearance of the elastic neutron scattering signal when measuring with energy analysis on a triple-axis instrument.

The top panel of Fig. 9 shows the elastic magnetic signal when integrated over the energy resolution of BSS, RITAII and DMC, respectively. With the resolution of BSS of $\sim 1 \mu \mathrm{eV}$, there is almost no elastic signal at temperatures above $\sim 100 \mathrm{~K}$. At RITA, the elastic signal at $300 \mathrm{~K}$ has decreased to approximately $20 \%$ of the low-temperature signal, while within the DMC resolution, most of the signal is still seen as elastic at $300 \mathrm{~K}$.
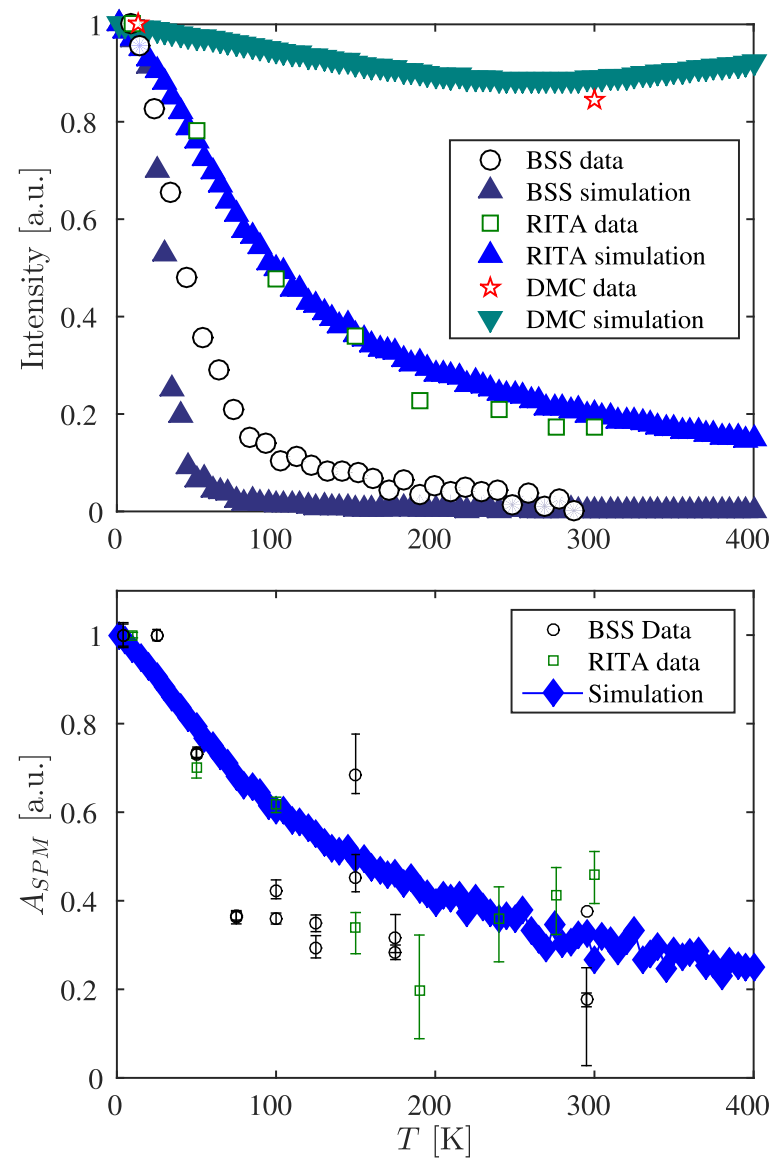

FIG. 9. (Color online) Temperature dependence of the elastic part of the inelastic neutron scattering signal. (Top) Background subtracted diffraction data from the backscattering spectrometer BSS, the triple-axis spectrometer RITA-2 and the diffractometer DMC. (Bottom) The area of the quasielastic superparamagnetic signal as obtained from modeling the data from BSS and RITA-2.

Overlayed on this, Fig. 9 shows the corresponding intensities from the numerical simulations, scaled only by a common factor. The agreement between experiment and simulation is striking for the DMC and RITA-2 data, even though $A_{\text {SPM }}$ was not used in the tuning of the simulations. This gives additional confidence in the simulated model. In the backscattering data, the experimental value of the elastic signal is in general higher than the simulations. This may well be a combination of two effects: (a) the difference in $\tau_{0}$ value, (b) a distribution of particle sizes, and therefore anisotropy barriers. Neither of these effects are included in the numerical simulations.

The bottom panel of Fig. 9 shows the total area of the fitted quasielastic magnetic signal, $A_{\mathrm{SPM}}$ as a function of temperature. The fitted inelastic signal has been subtracted from the data, which should thus be equal for both the BSS and RITA experiment, which is indeed the case within the uncertainties. Agreement with the simulations is also found in this case.

\section{E. Perspectives of the rotor mode}

Our analytical and numerical results provide a qualitative explanation of the experimentally observed increase of $\omega_{\alpha}$ : 
when thermal excitations cause a canting of the sublattice spins out of the basal plane, a torque from the exchange interaction will appear. This dominates the axial anisotropy, $B_{2}$, and causes the spins to perform coherent precessions within the easy plane, hence inducing periodic reversals of $\hat{S}^{z}$. The frequency of the precession depends on the canting, and hence on temperature. This displaces the neutron scattering signal from (quasi-)elastic to inelastic, in accordance with experimental observation. We speculate that the rotor mode may be present in other nanoparticles with a strong planar anisotropy, e.g., $\mathrm{NiO}$, although the temperature effect in this system was found to be less pronounced [14].

\section{CONCLUSION}

We observe an increase with temperature of the mean frequency of the uniform mode in weakly interacting antiferromagnetic 8-nm hematite particles. Simultaneously, we observe a disappearance of the strictly elastic signal from static magnetic order. Numerical Langevin simulations of a fairly detailed model are able to reproduce the features of the experimental data with sufficient accuracy. In combination with an analytical model, the simulations show that both observations can be understood by the presence of a fast dynamical mode-the "rotor" mode, which can be seen as a coherent superparamagnetic relaxation. In contrast to other uniform modes in magnetic nanoparticles, the rotor mode is driven by temperature-induced canting of the sublattice spins and causes a periodic magnetization reversal that transforms the spin system to become purely dynamical. Our results are in good quantitative agreement with earlier neutron scattering and Mössbauer data. We predict this mode to be of general relevance for antiferromagnetic nanoparticles with strong easy-plane anisotropy.

\section{ACKNOWLEDGMENTS}

We thank F. Bødker for preparing the nanoparticles and P.-A. Lindgård for stimulating discussions. A large thank goes to S. Mørup and C. Frandsen for participating in the initial phases of this project. This work was supported by the Danish Council for Independent Research though the Instrument Center DANSCATT. This work is based on neutron scattering experiments performed at SINQ, Paul Scherrer Institute, Switzerland, and at FRJ, Research Center Jülich, Germany.
[1] A. H. Lu, E. L. Salabas, and F. Schuth, Angew. Chem. Int. Ed. 46, 1222 (2007).

[2] S. Mørup, M. F. Hansen, and C. Frandsen, in Comprehensive Nanoscience and Technology, edited by D. Andrews, G. Scholes, and G. Wiederrecht (Elsevier, Amsterdam, 2011), Vol. 2, p. 437.

[3] L. Néel, Ann. Geophys. 5, 99 (1949).

[4] W. F. Brown Jr., Phys. Rev. 130, 1677 (1963).

[5] S. Mørup and H. Topsøe, Appl. Phys. 11, 63 (1976).

[6] S. Mørup, D. E. Madsen, C. Frandsen, C. R. H. Bahl, and M. F. Hansen, J. Phys.: Condens. Matter 19, 213202 (2007).

[7] C. R. H. Bahl, J. Garde, K. Lefmann, T. B. S. Jensen, D. E. Madsen, P.-A. Lindgård, and S. Mørup, Eur. J. Phys. B 62, 53 (2008).

[8] M. Hennion, C. Bellouard, J. Mirebeau, J. L. Dormann, and M. Nogues, Europhys. Lett. 25, 43 (1994).

[9] M. F. Hansen, F. Bødker, S. Mørup, K. Lefmann, K. N. Clausen, and P.-A. Lindgård, Phys. Rev. Lett. 79, 4910 (1997).

[10] K. Lefmann, F. Bødker, S. N. Klausen, M. F. Hansen, K. N. Clausen, P.-A. Lindgård, and S. Mørup, Europhys. Lett. 54, 526 (2001).

[11] S. N. Klausen, K. Lefmann, P.-A. Lindgård, K. N. Clausen, M. F. Hansen, F. Bødker, S. Mørup, and M. Telling, J. Magn. Magn. Mater. 266, 68 (2003).

[12] S. N. Klausen, K. Lefmann, P.-A. Lindgård, L. Theil Kuhn, C. R. H. Bahl, C. Frandsen, S. Mørup, B. Roessli, N. Cavadini, and Ch. Niedermayer, Phys. Rev. B 70, 214411 (2004).

[13] L. Theil Kuhn, K. Lefmann, C. R. H. Bahl, S. N. Ancona, P.-A. Lindgård, C. Frandsen, D. E. Madsen, and S. Mørup, Phys. Rev. B 74, 184406 (2006).

[14] C. R. H. Bahl, K. Lefmann, L. Theil Kuhn, N. B. Christensen, H. Vazquez, and S. Mørup, J. Phys.: Condens. Matter 18, 11203 (2006).
[15] M. Feygenson, X. Teng, S. E. Inderhees, Y. Yiu, W. Du, W. Han, J. Wen, Z. Xu, A. A. Podlesnyak, J. L. Niedziela, M. Hagen, Y. Qiu, C. M. Brown, L. Zhang, and M. C. Aronson, Phys. Rev. B 83, 174414 (2011)

[16] S. Disch, R. P. Hermann, E. Wetterskog, A. A. Podlesnyak, K. An, T. Hyeon, G. Salazar-Alvarez, L. Bergström, and Th. Brückel, Phys. Rev. B 89, 064402 (2014).

[17] A. H. Hill, H. Jacobsen, J. R. Stewart, Feng Jiao, N. P. Jensen, S. L. Holm, H. Mutka, T. Seydel, A. Harrison, and K. Lefmann, J. Chem. Phys. 140, 044709 (2014).

[18] E. Brok, C. Frandsen, D. E. Madsen, H. Jacobsen, J. O. Birk, K. Lefmann, J. Bendix, K. S. Pedersen, C. B. Boothroyd, A. A. Berhe, G. G. Simeoni, and S. Mørup, J. Phys. D 47, 365003 (2014).

[19] A. H. Morrish, Canted Antiferromagnetism: Hematite (World Scientific, Singapore, 1994).

[20] W. Kündig, H. Bömmel, G. Constabaris, and R. H. Lindquist, Phys. Rev. 142, 137 (1966).

[21] H. Jacobsen, J. Garde, P. Hedegård, and K. Lefmann (unpublished).

[22] C. R. H. Bahl, P. Andersen, S. N. Klausen, and K. Lefmann, Nucl. Instr. Meth. B 226, 667 (2004).

[23] C. R. H. Bahl, K. Lefmann, A. B. Abrahamsen, H. M. Rønnow, F. Saxild, T. B. S. Jensen, L. Udby, N. H. Andersen, N. B. Christensen, H. S. Jacobsen, T. Larsen, P. Häfliger, S. Streule, and Ch. Niedermayer, Nucl. Instr. Meth. B 246, 452 (2006).

[24] W. H. Press, S. A. Teukolsky, W. H. Vetterling, and B. P. Flannery, Numerical Recipes in C (Cambridge University Press, Cambridge, UK, 2007).

[25] A. Würger, Europhys. Lett. 44, 103 (1998).

[26] M. F. Hansen, F. Bødker, S. Mørup, K. Lefmann, K. N. Clausen, and P.-A. Lindgård, J. Magn. Magn. Mater. 221, 10 (2000). 
[27] H. Jacobsen, M.Sc. thesis, Niels Bohr Institute, University of Copenhagen, 2014.

[28] E. J. Samuelsen and G. Shirane, Phys. Stat. Sol. 42, 241 (1970).

[29] J. L. García-Palacios and F. J. Lázaro, Phys. Rev. B 58, 14937 (1998).
[30] L. D. Landau and E. M. Lifshitz, Statistical Physics, 3rd ed. (Butterworth-Heinemann, Boston, 1980).

[31] J. Garde, M.Sc. thesis, Niels Bohr Institute, University of Copenhagen, 2008.

[32] K. F. Riley, M. P. Hobson, and S. J. Bence, Mathematical Methods for Physics and Engineering (Cambridge University Press, Cambridge, UK, 2007). 